\title{
Aspirin use in relation to long-term survival after gastrectomy for gastric adenocarcinoma
}

\author{
Dag Holmberg ${ }^{1}$ (1) . Joonas H. Kauppila ${ }^{1,2}$. Fredrik Mattsson ${ }^{1}$. Johannes Asplund ${ }^{1}$. Wilhelm Leijonmarck ${ }^{1}$. \\ Shao-Hua Xie ${ }^{1}$. Jesper Lagergren ${ }^{1,3}$
}

Received: 4 November 2021 / Accepted: 28 January 2022 / Published online: 15 February 2022

(c) The Author(s) 2022

\begin{abstract}
Background Low-dose aspirin use may reduce cancer incidence and mortality, but its influence on gastric adenocarcinoma survival is unclear. This study aimed to assess whether aspirin use improves long-term survival following gastrectomy for gastric adenocarcinoma.

Methods This population-based cohort study included almost all patients who underwent gastrectomy for gastric adenocarcinoma in Sweden from 2006 to 2015, with follow-up throughout 2020. Preoperative exposure to a daily low-dose (75-160 mg) aspirin for 1 (main exposure), 2 and 3 years and for 1 year after gastrectomy was examined in relation to 5-year all-cause mortality (primary outcome) and disease-specific mortality. Multivariable Cox regression provided hazard ratios (HR) with 95\% confidence intervals (CI), adjusted for age, sex, education, calendar year, comorbidity, statin use, tumour location, tumour stage, neoadjuvant chemotherapy, surgeon volume of gastrectomy and surgical radicality.

Results Among 2025 patients, 545 (26.9\%) used aspirin at the date of gastrectomy. Aspirin use within 1 year before surgery did not decrease the adjusted risk of 5-year all-cause mortality $(\mathrm{HR}=0.98,95 \% \mathrm{CI} 0.85-1.13)$ or disease-specific mortality $(\mathrm{HR}=1.00,95 \% \mathrm{CI} 0.86-1.17)$. Preoperative aspirin use for 2 years $(\mathrm{HR}=0.98,95 \% \mathrm{CI} 0.84-1.15)$ or 3 years $(\mathrm{HR}=0.94$, $95 \%$ CI 0.79-1.12) did not decrease the risk of 5-year all-cause mortality. Patients remaining on aspirin during the first year after gastrectomy had a similar 5-year all-cause mortality as non-users of aspirin (HR $=1.01,95 \%$ CI $0.82-1.25)$.

Conclusions Low-dose aspirin use might not improve long-term survival after gastrectomy for gastric adenocarcinoma and may thus not be a target for adjuvant therapy in this group of patients.
\end{abstract}

Keywords Aspirin · Chemoprevention · Gastric neoplasm · Gastrectomy

\section{Introduction}

Gastric cancer ( $>95 \%$ adenocarcinoma) is characterized by high incidence and poor survival, making it the third most common cause of cancer deaths globally [1]. In Europe, the overall 5-year survival rate is approximately $25 \%[2,3]$.

\section{Dag Holmberg}

dag.holmberg@ki.se

1 Upper Gastrointestinal Surgery, Department of Molecular Medicine and Surgery, Karolinska Institutet and Karolinska University Hospital, Retzius Street 13A, 4thFloor, 17177 Stockholm, Sweden

2 Department of Surgery, Oulu University Hospital and University of Oulu, Oulu, Finland

3 School of Cancer and Pharmacological Sciences, King's College London, London, UK
Surgery with total or subtotal gastrectomy, with or without pre- or perioperative chemotherapy, is the main curatively intended treatment [4].

Low-dose aspirin is commonly prescribed to prevent cardio- and cerebrovascular disease, but may also prevent cancer development and improve survival in patients with some cancer types [5, 6]. Aspirin inhibits the formation of pro-inflammatory prostaglandins, a product of the cyclooxygenase (COX)-1 and -2 complexes, which have been implicated in the formation of several neoplasias [7, 8]. Aspirin has also been suggested to counteract tumour growth and metastases in adenomatous neoplasia, possibly through blocking the production of thromboxane A2 in platelets, which counteracts platelet aggregation and may thus reduce tumour spread $[9,10]$. These results have prompted the initiation of randomized clinical trials assessing aspirin as a novel adjuvant treatment to surgery in patients with certain 
cancer types. Regarding gastric adenocarcinoma specifically, observational studies have indicated that aspirin may reduce its incidence [11-13], but there is a paucity of data regarding a potential survival benefit of aspirin as an adjuvant therapy to gastrectomy in patients with this cancer.

This study set out to assess the hypothesis that preoperative and postoperative use of low-dose aspirin improves survival in patients who undergo gastrectomy for gastric adenocarcinoma.

\section{Methods}

\section{Design}

This was a nationwide Swedish population-based cohort study, titled the Swedish Gastric Cancer Surgery Study (SWEGASS), which has been presented in detail in a recent cohort description [14]. The cohort included $98 \%$ of all patients having undergone gastrectomy for gastric adenocarcinoma (including Siewert type III tumours of the gastric cardia) in Sweden from July 1, 2006 to December 31, 2015 and for the purpose of the present study, the follow-up was updated until December 31, 2020. Potentially eligible patients were initially identified in the Swedish Cancer Registry and Swedish Patient Registry by the disease, histopathology and surgery codes defining gastric adenocarcinoma and gastrectomy. The final cohort was then selected after a review of medical records from all patients, including notes from histopathology reports, multidisciplinary meetings, surgery and hospital discharge [14]. The study was approved by the Regional Ethical Review Board in Stockholm, Sweden (registration number 2017/141-31/2).

\section{Exposure}

The main exposure was a daily dispensation of low-dose aspirin (75-160 mg) within 1 year prior to surgery. There were three secondary exposures: aspirin use for 2 years prior to surgery, 3 years prior to surgery and during the first postoperative year. The data on aspirin use came from the Swedish Prescribed Drug Registry, which electronically and automatically records all prescribed and dispensed drugs in Sweden (except for in-hospital use). The registry is nearly $100 \%$ complete [15]. Low-dose aspirin is only available by prescription in Sweden.

\section{Outcomes}

The main outcome was 5-year all-cause mortality, defined as death from any cause occurring between the date of gastrectomy and 5 years postoperatively. The secondary outcome was 5-year disease-specific mortality, defined as death from gastric cancer as an underlying or contributing cause of death within 5 years of the gastrectomy. Information on the mortality outcomes was obtained from the Swedish Cause of Death Registry. This registry has $100 \%$ completeness for date of death and $96 \%$ completeness for cause of death in all Swedish residents and also includes deaths among Swedish residents who die abroad [16]. Because information on date of death is updated continuously, while causes of death are assembled at the end of each calendar year, follow-up for allcause mortality was one year longer (December 31, 2020) than for disease-specific mortality (December 31, 2019).

\section{Covariates}

We considered the following eleven covariates with categorizations in parenthesis: age (continuous), sex (male or female), education ( $\leq 9$ years, $10-12$ years or $>12$ years of formal education), calendar year (continuous), comorbidity (Charlson comorbidity index score 0,1 or $\geq 2$ ), statin use (yes or no), tumour location (cardia or non-cardia), pathological tumour stage (0-I, II, III or IV), neoadjuvant chemotherapy (yes or no), annual surgeon volume of gastrectomy (quartiles, i.e. four equal-sized groups) and radicality of the surgical resection (R0 or R1/R2). The data on age, sex, education, calendar year, comorbidity and statin use were obtained from three nationwide complete Swedish registries: Patient Registry, Longitudinal Integrated Database for Health Insurance and Labour Market Studies (LISA) and Prescribed Drug Registry [15, 17, 18]. Comorbidity was classified based on the most well-validated version of the Charlson comorbidity index [19]. Statin use was included as a covariate since it is often used alongside aspirin and may improve gastric cancer survival [20, 21]. Information on tumour location, tumour stage, neoadjuvant chemotherapy, surgeon volume and surgical radicality was retrieved from a review of medical records.

\section{Statistical analysis}

The study patients were followed up from the date of gastrectomy until death, 5 years after surgery or end of the study period, whichever occurred first. The cumulative survival as a function of time was estimated using the Kaplan-Meier estimator for a descriptive comparison of users and non-users of aspirin. Cox proportional hazards models were used to calculate hazard ratios (HR) with $95 \%$ confidence intervals (CI), comparing the hazard rates of mortality in aspirin users with non-users of aspirin (reference group in all analyses). A multivariable model was adjusted for the eleven covariates and categorizations presented above. In a sensitivity analysis, the study cohort was restricted to patients with curatively intended treatment. To further evaluate whether potential associations 
between exposure and outcome were modified by covariates, an interaction term was included in the models. HRs with $95 \%$ CI were derived within each stratum for age ( $\leq 66,67-76$ and $\geq 77$ years), sex (male or female), comorbidity (Charlson comorbidity index score 0,1 and $\geq 2$ ) and tumour stage (0-I, II, III and IV). This was done for each covariate separately. The missing data were found in at least one of the eleven covariates in $12 \%$ of patients. To manage missing data, both multiple imputation and complete case analyses were conducted. In the multiple imputation analysis, the number of imputed datasets were 20 and the monotone logistic method in PROC MI was used with the assumption that missing occurred at random [22]. Imputation was conducted separately for the two outcomes and included the eleven covariates in the multivariable model. PROC MIANALYZE was used to combine the results from the analyses of the 20 datasets. Because the results from the multiple imputation analysis were considered less prone to bias and the results were similar to those of the complete case analysis, we only present the results from the multiple imputation. The proportional hazards assumption was evaluated using log-log survival plots and by calculating the correlations between Schoenfeld residuals for a particular covariate and ranking of individual failure time. The correlations were low, indicating that the proportional hazards assumption was met for all analyses. A senior biostatistician (FM) conducted the data management and statistical analyses according to a detailed and pre-defined study protocol and used the statistical software SAS/STAT Statistical Package, Version 9.4 (SAS Institute Inc., Cary, NC, USA) for these purposes.

\section{Results}

\section{Patients}

The study included 2025 patients who underwent gastrectomy for gastric adenocarcinoma and they contributed a total of 5684 person-years and a mean of 2.8 person-years. Of these patients, $545(26.9 \%)$ patients used aspirin at the time of surgery. Among the aspirin users, 178 (32.7\%) did not dispense any further aspirin after surgery. Some $56(2.8 \%)$ patients started using aspirin within one year of gastrectomy. Aspirin users were generally older, more often men, had lower education, more comorbidities, more statin use, more severe complications (Clavien Dindo $\geq 3$ ), lower rate of neoadjuvant chemotherapy and a higher rate of sub-total compared to total gastrectomy, whereas other variables were more similarly distributed (Table 1). Mortality within 90 days of surgery occurred in $52(9.5 \%)$ of the aspirin users and $91(6.2 \%)$ of the non-users of aspirin.
Table 1 Characteristics of 2,025 patients who underwent gastrectomy for gastric adenocarcinoma

\begin{tabular}{|c|c|c|}
\hline & \multicolumn{2}{|l|}{ Number $(\%)$} \\
\hline & Aspirin users & Non-users of aspirin \\
\hline Total & $545(100.0)$ & $1480(100.0)$ \\
\hline Mean age (standard deviation) & $75.4(8.2)$ & $67.6(12.0)$ \\
\hline \multicolumn{3}{|l|}{ Sex } \\
\hline Men & $354(65.0)$ & $813(54.9)$ \\
\hline Women & $191(35.0)$ & $667(45.1)$ \\
\hline \multicolumn{3}{|l|}{ Education level (years) } \\
\hline$\leq 9$ & $269(49.4)$ & $561(37.9)$ \\
\hline $10-12$ & $201(36.9)$ & $603(40.7)$ \\
\hline$>12$ & $58(10.6)$ & $286(19.3)$ \\
\hline Not known & $17(3.1)$ & $30(2.0)$ \\
\hline \multicolumn{3}{|l|}{ Calendar period } \\
\hline$<2011$ & $293(53.8)$ & $753(50.9)$ \\
\hline$\geq 2011$ & $252(46.2)$ & $727(49.1)$ \\
\hline \multicolumn{3}{|l|}{ Charlson comorbidity index } \\
\hline 0 & $119(21.8)$ & $742(50.1)$ \\
\hline 1 & 185 (33.9) & $476(32.2)$ \\
\hline$\geq 2$ & $241(44.2)$ & $262(17.7)$ \\
\hline \multicolumn{3}{|l|}{ Statin use } \\
\hline Yes & $263(48.3)$ & $168(11.3)$ \\
\hline No & $282(51.7)$ & $1,312(88.7)$ \\
\hline \multicolumn{3}{|l|}{ Tumour localization } \\
\hline Cardia & $59(10.8)$ & $169(11.4)$ \\
\hline Non-cardia & $481(88.3)$ & $1,304(88.1)$ \\
\hline Not known & $5(0.9)$ & $7(0.5)$ \\
\hline \multicolumn{3}{|l|}{ Tumour stage } \\
\hline $0-\mathrm{I}$ & $149(27.3)$ & $337(22.8)$ \\
\hline II & $157(28.8)$ & $419(28.3)$ \\
\hline III & $182(33.4)$ & $529(35.7)$ \\
\hline IV & $40(7.3)$ & $155(10.5)$ \\
\hline Not known & $17(3.1)$ & $40(2.7)$ \\
\hline \multicolumn{3}{|l|}{ Histological subtype } \\
\hline Diffuse & $152(27.9)$ & $537(36.3)$ \\
\hline Intestinal & $204(37.4)$ & 469 (31.7) \\
\hline Mixed & $17(3.1)$ & $61(4.1)$ \\
\hline Indeterminate & $1(0.2)$ & $8(0.5)$ \\
\hline Not known & $171(31.4)$ & $405(27.4)$ \\
\hline \multicolumn{3}{|l|}{ Neoadjuvant chemotherapy } \\
\hline Yes & $80(14.7)$ & $510(34.5)$ \\
\hline No & $459(84.2)$ & $960(64.9)$ \\
\hline Not known & $6(1.1)$ & $10(0.6)$ \\
\hline \multicolumn{3}{|l|}{ Type of gastrectomy } \\
\hline Total gastrectomy & $199(36.5)$ & $686(46.4)$ \\
\hline Subtotal gastrectomy & $322(59.1)$ & $747(50.5)$ \\
\hline Not known & $24(4.4)$ & $47(3.1)$ \\
\hline \multicolumn{3}{|l|}{ Surgeon volume } \\
\hline$<2.3$ & $146(26.8)$ & $360(24.3)$ \\
\hline $2.3-3.9$ & $146(26.8)$ & $358(24.2)$ \\
\hline $4.0-5.7$ & $133(24.4)$ & 365 (24.7) \\
\hline
\end{tabular}


Table 1 (continued)

\begin{tabular}{lcc}
\hline & \multicolumn{2}{c}{ Number $(\%)$} \\
\cline { 2 - 3 } & Aspirin users & Non-users of aspirin \\
\hline$>5.7$ & $117(21.5)$ & $386(26.1)$ \\
$\quad$ Not known & $3(0.6)$ & $11(0.7)$ \\
Surgical radicality & & \\
Yes (R0) & $452(82.9)$ & $1,198(80.9)$ \\
No (R1) & $63(11.6)$ & $183(12.4)$ \\
Not known & $30(5.5)$ & $99(6.7)$ \\
Postoperative complications & & \\
$\quad$ Clavien-Dindo) & & \\
None & $305(56.0)$ & $917(62.0)$ \\
I & $21(3.9)$ & $32(2.2)$ \\
II & $94(17.2)$ & $270(18.2)$ \\
$\geq$ III & $125(22.9)$ & $261(17.6)$ \\
\hline
\end{tabular}

\section{Aspirin use and risk of mortality}

The Kaplan-Meier curves were similar comparing users and non-users of aspirin (Fig. 1). Aspirin use within 1 year prior to gastrectomy was not associated with any decreased risk of 5-year all-cause mortality (adjusted HR 0.98, 95\% CI $0.85-1.13$ ) or 5-year disease-specific mortality (adjusted HR 1.00, 95\% CI 0.86-1.17) (Table 2). Stratified analyses showed similar results across age groups, sexes, comorbidity scores and tumour stages (Table 2). Patients using daily aspirin for 2 and 3 years prior to surgery did not show any decreased adjusted HRs of 5-year all-cause mortality (HR $0.98,95 \%$ CI $0.84-1.15$, for 2 years and HR $0.94,95 \%$ CI $0.79-1.12$, for 3 years). Likewise, patients using aspirin both preoperatively and for 1 year postoperatively did not show any decreased risk of 5-year all-cause mortality (adjusted HR $1.01,95 \%$ CI 0.82-1.25).

\section{Sensitivity analysis}

In a sensitivity analysis, we excluded 291 (14.3\%) patients who underwent gastrectomy without a clearly curative intent. In this analysis of 1,734 patients, the 90-day mortality rate was $4.0 \%$. Aspirin use was not associated with either 5-year all-cause mortality (adjusted HR 0.92, 95\% CI $0.78-1.09$ ) or 5-year disease-specific mortality (adjusted HR $0.94,95 \%$ CI $0.79-1.13$ ), compared to non-users.

\section{Discussion}

This study found no support for the hypothesis that aspirin use improves the 5-year survival in patients who undergo gastrectomy for gastric adenocarcinoma. These null results were consistent across ages, sexes, comorbidity scores, tumour stages, lengths of preoperative use of aspirin and in patients who continued using aspirin after gastrectomy, as well as in patients who underwent clearly curatively intended surgery.

Methodological strengths of this study include the population-based design with full national coverage, complete and long-term follow-up of all patients, large cohort size, prospectively collected and detailed clinical data with low proportions of missing and adjustment for all established and several potential prognostic factors. The information on the exposures (aspirin use), outcomes (5-year mortality) and covariates was accurate. Only adenocarcinoma was included because other histological types of gastric malignancies may
Fig. 1 Survival probability following curatively intended gastrectomy for gastric adenocarcinoma among users of aspirin versus non-users of aspirin

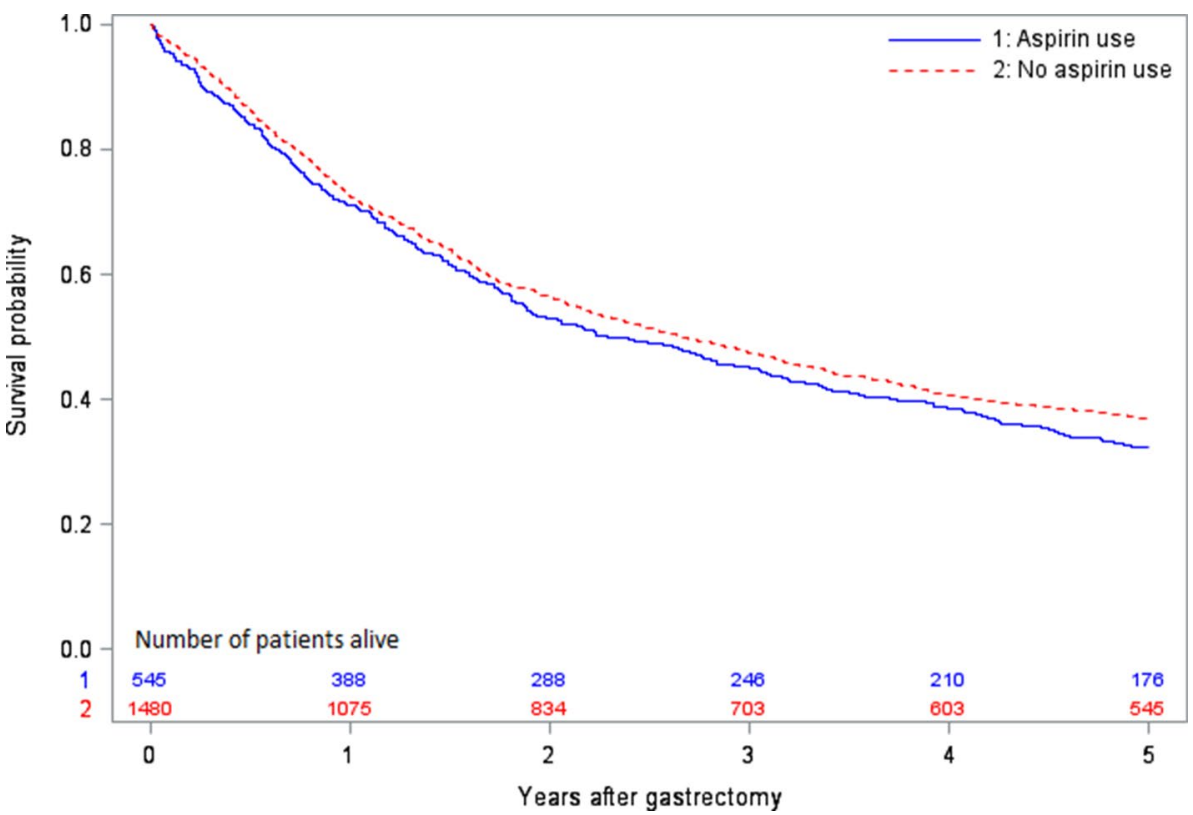


Table 2 Aspirin use and risk of 5-year all-cause and disease-specific mortality among 2,025 patients who underwent gastrectomy for gastric adenocarcinoma

\begin{tabular}{|c|c|c|c|c|c|c|}
\hline \multirow[b]{2}{*}{ At risk (n) } & \multirow[b]{2}{*}{ Person-years } & & \multicolumn{2}{|c|}{ All-cause mortality } & \multicolumn{2}{|c|}{ Disease-specific mortality } \\
\hline & & Deaths (n) & $\begin{array}{l}\text { Unadjusted HR } \\
(95 \% \mathrm{CI})\end{array}$ & $\begin{array}{l}\text { Adjusted HR } \\
(95 \% \mathrm{CI})\end{array}$ & $\begin{array}{l}\text { Unadjusted HR } \\
(95 \% \mathrm{CI})\end{array}$ & $\begin{array}{l}\text { Adjusted HR } \\
(95 \% \mathrm{CI})\end{array}$ \\
\hline
\end{tabular}

\section{Overall}

Aspirin

No

Yes

$1,480 \quad 4,210$

$545 \quad 1,478$

$\leq 66$ years

Aspirin

No

Yes

$614 \quad 1,965$

79

242

67-76 years

Aspirin

No

Yes

$\geq 77$ years

Aspirin

No

Yes

Men

Aspirin

No

Yes

Women

Aspirin

No

Yes

No comorbidity

Aspirin

No

Yes

197

557

314

123

344

45

1.00 (reference)

1.06 (0.77-1.44)

1.00 (reference)

1.00 (reference)

1.04 (0.75-1.45)

1.00 (reference)

1.04 (0.75-1.44)

1.00 (reference)

1.05 (0.92-1.20)

1.00 (reference)

$1.00(0.86-1.17)$

$0.98(0.85-1.13)$

1.00 (reference)

1.00 (reference)

1.00 (reference)

1.00 (reference)

0.95 (0.77-1.17)

$0.93(0.75-1.17)$

$0.89(0.71-1.11)$

$0.91(0.82-1.16)$

899

277

201

1.00 (reference)

1.00 (reference)

1.00 (reference)

1.00 (reference)

0.97 (0.81-1.16)

1.02 (0.84-1.24)

$0.98(0.80-1.20)$

1.07 (0.86-1.33)

1 comorbidity

Aspirin

No

Yes

1,356

2,321

424

73

1.00 (reference)

$1.14(0.89-1.46)$

1.00 (reference)

$1.12(0.87-1.45)$

1.00 (reference)

1.05 (0.80-1.37)

1.00 (reference)

$1.11(0.84-1.47)$

$\geq 2$ comorbidities

Aspirin

No

Yes

Tumour stage 0-I

Aspirin

No

Yes

$337 \quad 1,457$

557

80

62

206

181

1.00 (reference)

1.00 (reference)

(reference)

1.00 (reference)

$0.81(0.66-0.98)$

0.87 (0.70-1.07)

$0.77(0.62-0.97)$

$0.87(0.69-1.11)$

Tumour stage II

Aspirin

$\begin{array}{llllllll}\text { No } & 419 & 1,384 & 240 & 1.00 \text { (reference) } & 1.00 \text { (reference) } & 1.00 \text { (reference) } & 1.00(\text { reference) } \\ \text { Yes } & 157 & 517 & 98 & 1.08(0.86-1.36) & 0.80(0.63-1.03) & 1.05(0.82-1.36) & 0.85(0.64-1.11) \\ \begin{array}{l}\text { Tumour stage III } \\ \begin{array}{l}\text { Aspirin } \\ \text { No }\end{array}\end{array} & & & & & & & \\ \end{array}$

$\begin{array}{llll}1.00 \text { (reference) } & 1.00 \text { (reference) } & 1.00 \text { (reference) } & 1.00 \text { (reference) } \\ 1.92(1.38-2.67) & 1.46(1.04-2.05) & 1.78(1.14-2.78) & 1.45(0.92-2.28) \\ & & & \\ 1.00 \text { (reference) } & 1.00 \text { (reference) } & 1.00 \text { (reference) } & 1.00 \text { (reference) } \\ 1.08(0.86-1.36) & 0.80(0.63-1.03) & 1.05(0.82-1.36) & 0.85(0.64-1.11) \\ & & & \\ 1.00 \text { (reference) } & 1.00 \text { (reference) } & 1.00 \text { (reference) } & 1.00 \text { (reference) }\end{array}$


Table 2 (continued)

\begin{tabular}{|c|c|c|c|c|c|c|c|}
\hline & \multirow[b]{2}{*}{ At risk (n) } & \multirow[b]{2}{*}{ Person-years } & \multirow[b]{2}{*}{ Deaths (n) } & \multicolumn{2}{|c|}{ All-cause mortality } & \multicolumn{2}{|c|}{ Disease-specific mortality } \\
\hline & & & & $\begin{array}{l}\text { Unadjusted HR } \\
(95 \% \mathrm{CI})\end{array}$ & $\begin{array}{l}\text { Adjusted HR } \\
(95 \% \mathrm{CI})\end{array}$ & $\begin{array}{l}\text { Unadjusted HR } \\
(95 \% \mathrm{CI})\end{array}$ & $\begin{array}{l}\text { Adjusted HR } \\
(95 \% \mathrm{CI})\end{array}$ \\
\hline Yes & 182 & 316 & 158 & $1.17(0.98-1.40)$ & $0.88(0.72-1.07)$ & $1.15(0.96-1.39)$ & $0.93(0.75-1.15)$ \\
\hline \multicolumn{8}{|c|}{ Tumour stage IV } \\
\hline \multicolumn{8}{|c|}{ Aspirin } \\
\hline No & 155 & 234 & 139 & 1.00 (reference) & 1.00 (reference) & 1.00 (reference) & 1.00 (reference) \\
\hline Yes & 40 & 37 & 39 & $1.64(1.15-2.34)$ & $1.47(1.01-2.13)$ & $1.59(1.10-2.30)$ & $1.46(0.99-2.14)$ \\
\hline
\end{tabular}

*Adjusted for age, sex, education, calendar year, comorbidity, statin use, tumour location, tumour stage, neoadjuvant chemotherapy, surgeon volume and surgical radicality

have different treatment and survival. There are also weaknesses. Low-dose aspirin requires a prescription in Sweden, but small packages of high-dose aspirin are available overthe-counter, which might introduce limited misclassification of the exposure. The observational design is subject to unknown or residual confounding. Some exposures that could have confounded the findings are tobacco smoking, alcohol overconsumption and obesity. However, although these exposures were not directly adjusted for, they were still accounted for to some extent by the adjustments for comorbidities related to these exposures. Residual confounding by comorbidity related to aspirin use is also unlikely given that the null results observed for the all-cause mortality outcomes were confirmed in the analyses of gastric adenocarcinoma-specific mortality. Data on chemotherapyrelated fever were not available, but were unlikely to interact with aspirin use given that continuous use was required and low-dose aspirin is not prescribed as an anti-pyretic agent.

The role of low-dose aspirin as a potential anticarcinogenic agent has been studied intensively during the last decades. This research has been encouraged by the results of a long-term follow-up of five randomized clinical trials with 17,285 participants who randomly received aspirin or placebo/control medication for the prevention of cardiovascular disease [10]. In that study, aspirin users were at a $31 \%$ decreased relative risk of metastasis upon diagnosis of various adenocarcinoma sites (including the stomach) and among aspirin users with a localized tumour, the risk of subsequent metastasis was 55\% decreased [10]. However, the trial was not powered to examine gastric cancer separately. The evidence of a cancer-preventive effect of aspirin use has been strongest for colorectal cancer [23-25]. However, a recent randomized clinical trial showed a similar risk of mortality in users of celecoxib (a COX-2 inhibitor) and placebo in 2,524 patients with stage III colorectal cancer (HR $0.86,95 \%$ CI 0.72-1.04) [26].

Regarding gastric cancer specifically, a meta-analysis of 33 observational studies and 1,927,971 patients indicated that aspirin use slightly decreases the risk of developing this tumour, with pooled risk ratios in fixed- and random-effects models of 0.89 (95\% CI 0.87-0.91) and 0.83 (0.74-0.92), respectively [13]. Yet, only one previous study has examined whether aspirin use improves survival in patients with established gastric cancer. That was a study from the United Kingdom analysing two cohorts with a total of 1,720 patients who underwent surgery for gastric cancer and similar to the present study, aspirin use was not associated with any decreased mortality [27]. However, that study was confounded by more than $80 \%$ missing tumour stage, the most important prognostic factor of gastric cancer and included all histologies and also nonsurgical patients. An ongoing trial is investigating whether aspirin use decreases mortality in gastric and oesophageal cancer, but preliminary results are not expected until 2027 [28]. In the meantime, the findings from the current study together with the previous study provide the best available evidence and may prompt an interim analysis of the trial.

In conclusion, this population-based cohort study with complete follow-up and adjustment for all known prognostic factors indicates that the use of aspirin does not decrease the all-cause or 5-year disease-specific mortality in patients who undergo curatively intended gastrectomy for gastric adenocarcinoma.

Funding Open access funding provided by Karolinska Institutet. The study was supported by the Swedish Research Council, Swedish Cancer Society, and Stockholm Cancer Society.

Data availability statement The data that support the findings of this study are available from The Swedish Board of Health and Welfare. Restrictions apply to the availability of these data, which were used under license for this study. The data are available from the authors with the permission of The Swedish Board of Health and Welfare.

\section{Declarations}

Conflict of interest The authors declare no conflict of interest. 
Open Access This article is licensed under a Creative Commons Attribution 4.0 International License, which permits use, sharing, adaptation, distribution and reproduction in any medium or format, as long as you give appropriate credit to the original author(s) and the source, provide a link to the Creative Commons licence, and indicate if changes were made. The images or other third party material in this article are included in the article's Creative Commons licence, unless indicated otherwise in a credit line to the material. If material is not included in the article's Creative Commons licence and your intended use is not permitted by statutory regulation or exceeds the permitted use, you will need to obtain permission directly from the copyright holder. To view a copy of this licence, visit http://creativecommons.org/licenses/by/4.0/.

\section{References}

1. Sung H, Ferlay J, Siegel RL, et al. Global cancer statistics 2020: GLOBOCAN estimates of incidence and mortality worldwide for 36 cancers in 185 countries. CA Cancer J Clin. 2021;71(3):209-49.

2. Fitzmaurice C, Abate D, Abbasi N, et al. Global, regional, and national cancer incidence, mortality, years of life lost, years lived with disability, and disability-adjusted life-years for 29 cancer groups, 1990 to 2017: a systematic analysis for the global burden of disease study. JAMA Oncol. 2019;5(12):1749-68. https://doi. org/10.1001/jamaoncol.2019.2996.

3. Anderson LA, Tavilla A, Brenner H, et al. Survival for oesophageal, stomach and small intestine cancers in Europe 1999-2007: results from EUROCARE-5. Eur J Cancer. 2015;51(15):2144-57. https://doi.org/10.1016/j.ejca.2015.07.026.

4. Smyth EC, Nilsson M, Grabsch HI, et al. Gastric cancer. Lancet. 2020;396(10251):635-48.

5. Algra AM, Rothwell PM. Effects of regular aspirin on long-term cancer incidence and metastasis: a systematic comparison of evidence from observational studies versus randomised trials. Lancet Oncol. 2012;13(5):518-27. https://doi.org/10.1016/S14702045(12)70112-2

6. Simon TG, Duberg A-S, Aleman S, et al. Association of aspirin with hepatocellular carcinoma and liver-related mortality. N Engl J Med. 2020;382(11):1018-28.

7. Thiel A, Mrena J, Ristimäki A. Cyclooxygenase-2 and gastric cancer. Cancer Metastasis Rev. 2011;20(3-4):387-95.

8. Liao LM, Vaughan TL, Corley DA, et al. Nonsteroidal antiinflammatory drug use reduces risk of adenocarcinomas of the esophagus and esophagogastric junction in a pooled analysis. Gastroenterology. 2012;142(3):442-52. https://doi.org/10.1053/j. gastro.2011.11.019.

9. Rothwell PM, Fowkes FGR, Belch JFF, et al. Effect of daily aspirin on long-term risk of death due to cancer: analysis of individual patient data from randomised trials. Lancet. 2011;377(9759):3141. https://doi.org/10.1016/s0140-6736(10)62110-1.

10. Rothwell PM, Wilson M, Price JF, et al. Effect of daily aspirin on risk of cancer metastasis: a study of incident cancers during randomised controlled trials. Lancet. 2012;379(9826):1591-601. https://doi.org/10.1016/S0140-6736(12)60209-8.

11. Cheung KS, Chan EW, Wong AYS, et al. Aspirin and risk of gastric cancer after Helicobacter pylori eradication: a territorywide study. J Natl Cancer Inst. 2018;110(7):743. https://doi.org/ 10.1093/jnci/djx267.

12. Kim JM-H, Chang MJ, Kim MW, et al. Cumulative dose threshold for the chemopreventive effect of aspirin against gastric cancer. Am J Gastroenterol. 2018;113(6):845-54. https://doi.org/10.1038/ s41395-018-0097-5.

13. Niikura R, Hirata Y, Hayakawa Y, et al. Effect of aspirin use on gastric cancer incidence and survival: a systematic review and meta-analysis. JGH open. 2020;4(2):117-25. https://doi.org/10. 1002/jgh3.12226.

14. Asplund J, Gottlieb-Vedi E, Leijonmarck W, et al. Prognosis after surgery for gastric adenocarcinoma in the Swedish Gastric Cancer Surgery Study (SWEGASS). Acta Oncol. 2021;60(4):513-20.

15. Wettermark B, Hammar N, Fored CM, et al. The new Swedish Prescribed Drug Register-opportunities for pharmacoepidemiological research and experience from the first six months. Pharmacoepidemiol Drug Saf. 2007;16(7):726-35. https://doi.org/10. 1002/pds.1294.

16. Brooke H, Talbäck M, Hörnblad J, et al. The Swedish cause of death register. Eur J Epidemiol. 2017;32(9):765-73. https://doi. org/10.1007/s10654-017-0316-1.

17. Ludvigsson JF, Svedberg P, Olén O, et al. The longitudinal integrated database for health insurance and labour market studies (LISA) and its use in medical research. Eur J Epidemiol. 2019;34(4):423-37.

18. Ludvigsson JF, Andersson E, Ekbom A, et al. External review and validation of the Swedish national inpatient register. BMC Public Health. 2011;11:1-16. https://doi.org/10.1186/1471-2458-11-450.

19. Brusselaers N, Lagergren J. The Charlson comorbidity index in registry-based research. Methods Inf Med. 2017;56(5):401-6. https://doi.org/10.3414/me17-01-0051.

20. Spence AD, Busby J, Hughes CM, et al. Statin use and survival in patients with gastric cancer in two independent population-based cohorts. Pharmacoepidemiol Drug Saf. 2019;28(4):460-70.

21. Chung H, Kim HJ, Jung HC, et al. Statins and metachronous recurrence after endoscopic resection of early gastric cancer: a nationwide Korean cohort study. Gastric Cancer. 2020;23(4):659-66.

22. Roderick JA, Little DBR. Statistical analysis with missing data. 3rd ed. Chichester: John Wiley and Sons Ltd.; 2019.

23. Bibbins-Domingo K. Aspirin use for the primary prevention of cardiovascular disease and colorectal cancer: US Preventive Services Task Force recommendation statement. Ann Internal Med. 2016;164(12):836-45.

24. Liao X, Lochhead P, Nishihara R, et al. Aspirin use, tumor PIK3CA mutation, and colorectal-cancer survival. N Engl J Med. 2012;367(17):1596-606. https://doi.org/10.1056/NEJMoa1207 756.

25. Emilsson L, Holme $\varnothing$, Bretthauer M, et al. Systematic review with meta-analysis: the comparative effectiveness of aspirin vs screening for colorectal cancer prevention. Aliment Pharmacol Ther. 2017;45(2):193-204. https://doi.org/10.1111/apt.13857.

26. Meyerhardt JA, Shi Q, Fuchs CS, et al. Effect of celecoxib vs placebo added to standard adjuvant therapy on disease-free survival among patients with stage III colon cancer. JAMA. 2021;325(13):1277-86.

27. Spence AD, Busby J, Johnston BT, et al. Low-dose aspirin use does not increase survival in 2 independent population-based cohorts of patients with esophageal or gastric cancer. Gastroenterology. 2018;154(4):849-60.e1. https://doi.org/10.1053/j.gastro. 2017.10.044.

28. Coyle C, Cafferty FH, Rowley S, et al. ADD-ASPIRIN: A phase III, double-blind, placebo controlled, randomised trial assessing the effects of aspirin on disease recurrence and survival after primary therapy in common non-metastatic solid tumours. Contemp Clin Trials. 2016;51:56-64.

Publisher's Note Springer Nature remains neutral with regard to jurisdictional claims in published maps and institutional affiliations. 